RI-3-02

\title{
A Note on Multi-trace Deformations and AdS/CFT
}

\author{
Amit Severi and Assaf Shomer? \\ Racah Institute of Physics, The Hebrew University, Jerusalem 91904, Israel \\ We derive the general formula, at a finite cutoff, for the change in the boundary \\ condition of a scalar field in AdS under a Multiple-trace deformation of the dual CFT. \\ Our analysis suggests that fluctuations around the classical solution in $A d S$ should not be \\ constrained by boundary conditions.
}

March 2002

1 E-mail : asever@cc.huji.ac.il.

2 E-mail : shomer@cc.huji.ac.il. 


\section{Introduction.}

The AdS/CFT correspondence [1,2, 3] claims that string theory on $A d S_{d+1} \times \mathcal{M}_{9-d}$ is dual to a $C F T_{d}$ that "lives" on the $d$-dimensional boundary of $A d S_{d+1}$. CFT operators that are dual to single particle states in string theory/gravity are usually referred to (in a language borrowed from 4-dimensional gauge theories) as "Single-trace" operators. Deformations of the CFT Lagrangian by such Single-trace operators are known to be related to deforming the dual string worldsheet by the corresponding vertex-operator. In the low energy gravity approximation this amounts to changing the vacuum expectation value of the dual gravity field (see [4,5] for review and references). Deforming the CFT Lagrangian by "Multi-trace" operators, corresponding to multi-particle states in the dual string/gravity picture, was recently argued [6,7] to give rise to a non-local generalization of the standard string theory worldsheet Lagrangian (NLST). In [8] the effect of a "Double-trace" deformation was analyzed in the gravity approximation and was shown to give rise to a change in the boundary condition to which the dual gravity field was subjected. An ansatz for the general case of "Multi-trace" deformations was given in [9] leading to the same conclusion. In this note we derive a general formula, at a finite cutoff, for the change in the boundary condition of a scalar field in AdS under a Multiple-trace deformation of the dual CFT. This is done using a formulation that is not the one usually used in the literature. Our result agrees with the one suggested in [9] when one removes the IR cutoff in AdS. The analysis we present raises the question whether or not one should impose boundary conditions in $A d S / C F T$. This point is discussed in section 4. Recent papers dealing with similar issues are $10,11,12]$.

\section{A finite cutoff formulation of the $A d S / C F T$ correspondence.}

In this section we summarize a finite cutoff formulation of the $A d S_{d+1} / C F T_{d}$ correspondence that was presented in [8] and which will be used in this note. This formulation is convenient for the discussion of Multi-trace deformations. We work in Euclidean $A d S_{d+1}$ in the Poincare patch with coordinates $(z, \vec{x})$ where the metric is given by (we set $R_{A d S}=1$ )

$$
d s^{2}=\frac{d z^{2}+d x_{i} d x_{j} \delta^{i j}}{z^{2}}
$$

The boundary of Euclidean $A d S$ in these coordinates is the surface $\partial(A d S) \equiv\{(z=0, \vec{x})\} \cup$ $\{(z=\infty,|\vec{x}|=\infty)\}$. We introduce a finite IR cutoff in $A d S$ by considering the surface 
$z=\epsilon<<1$. This introduces a problem near the special point in this parameterization $(z=\infty,|\vec{x}|=\infty)$ since as we approach $|\vec{x}| \rightarrow \infty$ the surface $z=\epsilon$ approaches the boundary (see figure 1). This is an artifact of our choice of coordinates. To avoid this problem we restrict all sources away from $\infty$. Taking that into account we denote the boundary of $A d S$ space at finite cutoff by $\partial \equiv\{(z=\epsilon, \vec{x})\}$ and the bulk by $\mathcal{B} \equiv\{(z>\epsilon, \vec{x})\}$.

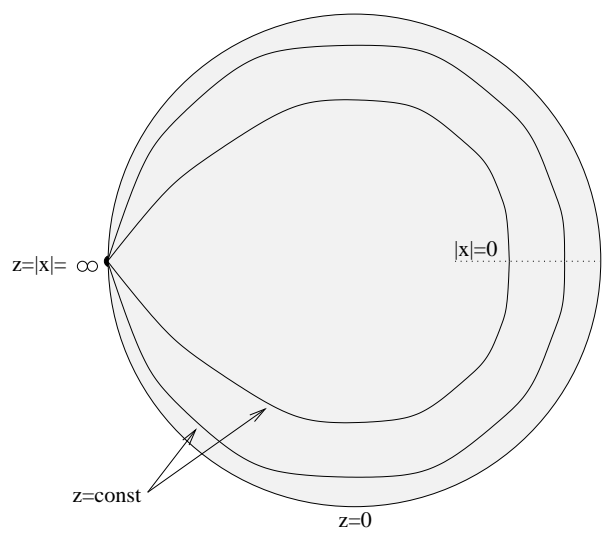

Figure 1: AdS space in Poincare coordinates.

We focus on the case of a scalar field in $A d S$, with mass $m^{2} \geq-\frac{d^{2}}{4}$, obeying the Breitenlohner Freedman bound and dual to a scalar CFT operator of dimension $\Delta$ where $\Delta(\Delta-d)=m^{2}$. We consider the following covariant gravity action which contains a boundary term that will prove important for the discussion of boundary conditions:

$$
\mathcal{S}_{0}[\phi]=\frac{1}{2} \int_{\mathcal{B}} d^{d+1} x \sqrt{g}\left[g^{\mu \nu} \partial_{\mu} \phi \partial_{\nu} \phi+m^{2} \phi^{2}\right]+\frac{\Delta}{2} \int_{\partial} \sqrt{\widehat{g}} \phi^{2},
$$

where $\widehat{g}$ stands for the metric restricted to the boundary. In Poincare coordinates the expression is:

$$
\mathcal{S}_{0}[\phi]=\frac{1}{2} \int_{\mathcal{B}} d^{d} x d z z^{-d+1}\left[\left(\partial_{z} \phi\right)^{2}+\left(\partial_{i} \phi\right)^{2}+\frac{m^{2}}{z^{2}} \phi^{2}\right]+\frac{\Delta}{2} \int_{\partial} \epsilon^{-d} \phi^{2} .
$$

The linear variation of this action is:

$$
\delta \mathcal{S}_{0}[\phi]=\int_{\mathcal{B}} d^{d+1} x \sqrt{g} \delta \phi\left[-\nabla^{2}+m^{2}\right] \phi-\int_{\partial} \epsilon^{-d} \delta \phi\left(z \partial_{z}-\Delta\right) \phi .
$$

In order to have classical solutions there are two choices. Either the field obeys a Dirichlet boundary condition $\left.\delta \phi\right|_{\partial}=0$, or it has to satisfy:

$$
\left.\left(z \partial_{z}-\Delta\right) \phi\right|_{\partial}=0
$$


This relation can be treated either as a boundary condition or as a boundary equation of motion without a boundary condition 3 . We adopt (2.5) in what follows and since the above mentioned ambiguity will be important later we will refer to (2.5) as a "boundary relation". In section 4 this issue will be discussed.

The solution to the bulk equation of motion $\left(\nabla^{2}-m^{2}\right) \phi=0$ is of the form [2]:

$$
\phi(\vec{x}, z)=\int d^{d} x^{\prime} \alpha\left(\vec{x}^{\prime}\right) \frac{z^{\Delta}}{\left(z^{2}+\left|\vec{x}-\vec{x}^{\prime}\right|^{2}\right)^{\Delta}},
$$

where $\frac{z^{\Delta}}{\left(z^{2}+\left|\vec{x}-\vec{x}^{\prime}\right|^{2}\right)^{\Delta}}$ is the "bulk-boundary" propagator. The meaning of (2.5) becomes clear when we consider the asymptotics of the classical solution near the boundary $z \rightarrow 0$.

$$
\phi(\vec{x}, z)=\left[\alpha(\vec{x}) z^{d-\Delta}+\beta(\vec{x}) z^{\Delta}\right]\left(1+\mathcal{O}\left(z^{2}\right)\right) .
$$

In order to get a finite result when $z \rightarrow 0$ we look at:

$$
z^{\Delta-d}\left(z \partial_{z}-\Delta\right) \phi(\vec{x}, z) \rightarrow(d-2 \Delta) \alpha(\vec{x})+0 \times \beta(\vec{x}) z^{2 \Delta-d}+\ldots
$$

The leading term is proportional to the boundary function $\alpha$ and demanding $z^{\Delta-d}\left(z \partial_{z}-\right.$ $\Delta) \phi(\vec{x}, z)=0$ amounts thus to the trivial solution $\phi(\vec{x}, z)=0$. To have a non trivial solution in this formulation one needs to change the action and correspondingly change (2.5). The way to do that is to check what boundary relation is obeyed by a general solution of the form (2.6):

$$
z^{\Delta-d}\left(z \partial_{z}-\Delta\right) \phi(\vec{x}, z)=-2 \Delta \int d^{d} x^{\prime} \alpha\left(\vec{x}^{\prime}\right) \frac{z^{2 \Delta-d+2}}{\left(z^{2}+\left|\vec{x}-\vec{x}^{\prime}\right|^{2}\right)^{\Delta+1}} \equiv(\mathbf{A} \alpha)(\vec{x}),
$$

where for brevity of our notation we denote this linear functional acting on $\alpha\left(\vec{x}^{\prime}\right)$ by $\mathbf{A}\left[\alpha\left(\vec{x}^{\prime}\right)\right](\vec{x})$. This cumbersome looking integral turns out to have very desirable properties as we will demonstrate shortly. In order to fix the boundary relation determined by some classical function $\rho(\vec{x})$ we thus write:

$$
\left.z^{\Delta-d}\left(z \partial_{z}-\Delta\right) \phi(\vec{x}, z)\right|_{\partial}=(\mathbf{A} \rho)(\vec{x}) .
$$

This fixes $\alpha(\vec{x})=\rho(\vec{x})$ and allows us to formulate conveniently the fact that the boundary relation sets the leading term in the expansion of the gravity field near the boundary of $\operatorname{AdS}[2,3]$, 13.

3 In the classical approximation the two are the same.

4 The sub leading term, $\beta$ parameterizes the expectation value of the dual CFT field $\mathcal{O}$ in the presence of a source term $\int \rho \mathcal{O}$. Regularity of the solution in Euclidean $A d S$ fixes this term as a function of the leading term $\alpha$. 
Moreover, as pointed out in (2.8), it is clear that (2.10) does not restrict terms which scale with $z$ like the subleading term $\beta(\vec{x}) z^{\Delta}$. In the Lorentzian version of the correspondence terms of this type are identified with the fluctuating $C F T$ degrees of freedom and it is thus desirable that they should not be constrained.

Note that, as usual in field theory, (2.2) subjected to (2.10) will not have nontrivial classical solutions unless we add to it a boundary source term to ensure that its linear variation vanishes also when $\rho \neq 0$. The action one gets in the presence of a source is thus:

$$
\mathcal{S}_{g r}[\phi ; \rho] \equiv \mathcal{S}_{0}[\phi]+\int_{\partial} d^{d} x d^{d} x^{\prime} \epsilon^{-\Delta} \phi(\vec{x}, z) \mathbf{A}\left(\vec{x}, \vec{x}^{\prime}\right) \rho\left(\vec{x}^{\prime}\right) \equiv \mathcal{S}_{0}[\phi]+\epsilon^{-\Delta} \phi \mathbf{A} \rho,
$$

since the vanishing of the linear variation:

$$
\delta \mathcal{S}_{g r}[\phi ; \rho]=\int_{\mathcal{B}} d^{d+1} x \sqrt{g} \delta \phi\left[-\nabla^{2}+m^{2}\right] \phi-\int_{\partial} \epsilon^{-\Delta} \delta \phi\left[z^{\Delta-d}\left(z \partial_{z}-\Delta\right) \phi-\mathbf{A} \rho\right],
$$

leads either to Dirichlet boundary condition or to the ones we use, namely (2.10) 5 .

The formulation of the $A d S / C F T$ correspondence [2,3] in terms of generating functionals in the classical gravity approximation is thus given by:

$$
Z[\rho]_{C F T} \equiv<e^{\int \rho \mathcal{O}}>_{C F T}=Z[\rho]_{\text {string }} \simeq \int D[\phi] e^{-\mathcal{S}_{g r}[\phi ; \rho]} \sim e^{-\mathcal{S}_{g r}\left[\phi_{\rho} ; \rho\right]},
$$

where $<\ldots>_{C F T}$ means the path integral in the CFT, the field $\phi$ is the gravity field dual to the CFT operator $\mathcal{O}$, and $\phi_{\rho}$ is the classical solution. Since we are interested only in the dynamics of one scalar field we slightly abused notations as if it is a field theory path integral over this scalar field. This is justified at low enough energies before quantum gravity and stringy effects set in, which is the regime we are interested in. Note that there are two ways to interpret this gravity path integral. Either the measure includes (2.10) as a boundary condition or that there is no boundary condition and (2.10) is imposed at the classical saddle point as a boundary equation of motion.

Let us first show that this reproduces the known results of $A d S / C F T$. Evaluating the action (2.11) on the classical solution (2.6) obeying (2.10), i.e. on

$$
\phi_{\rho}(\vec{x}, z)=\int d^{d} x^{\prime} \rho\left(\vec{x}^{\prime}\right) \frac{z^{\Delta}}{\left(z^{2}+\left|\vec{x}-\vec{x}^{\prime}\right|^{2}\right)^{\Delta}},
$$

5 Using (2.10) in (2.11) one gets the action we used in [8]. We will demonstrate below that the general analysis presented here reproduces the result in $[8]$. Note that the two actions are not equivalent if we choose Dirichlet boundary conditions. 
one gets at a finite cutoff $\epsilon$

$$
\mathcal{S}^{\epsilon}\left[\phi_{\rho} ; \rho\right]=\frac{1}{2} \int_{\partial} d^{d} x_{1} d^{d} x_{2} d^{d} x_{3} \frac{\rho\left(\vec{x}_{1}\right) \mathbf{A}\left(\overrightarrow{x_{2}}, \vec{x}_{3}\right) \rho\left(\vec{x}_{3}\right)}{\left(\epsilon^{2}+\left|\vec{x}_{1}-\vec{x}_{2}\right|^{2}\right)^{\Delta}}
$$

To remove the cutoff we use the formula (for $\gamma>\frac{d}{2}$ ):

$$
\lim _{z \rightarrow 0} \frac{z^{2 \gamma-d}}{\left(z^{2}+\left|\vec{x}-\vec{x}^{\prime}\right|^{2}\right)^{\gamma}}=\pi^{\frac{d}{2}} \frac{\Gamma\left(\gamma-\frac{d}{2}\right)}{\Gamma(\gamma)} \delta^{d}\left(\vec{x}-\vec{x}^{\prime}\right) .
$$

This gives (see (2.9) for the definition of $\mathbf{A}$ ):

$$
\lim _{z \rightarrow 0} \mathbf{A}\left(\vec{x}, \vec{x}^{\prime}\right)=\left(-2 \pi^{\frac{d}{2}}\left(\Delta-\frac{d}{2}\right) \frac{\Gamma\left(\Delta-\frac{d}{2}\right)}{\Gamma(\Delta)}\right) \delta^{d}\left(\vec{x}-\vec{x}^{\prime}\right)
$$

Thus after removing the cutoff the action becomes:

$$
\mathcal{S}\left[\phi_{\rho} ; \rho\right] \equiv \lim _{\epsilon \rightarrow 0} \mathcal{S}^{\epsilon}\left[\phi_{\rho} ; \rho\right]=-\left(\Delta-\frac{d}{2}\right) \pi^{\frac{d}{2}} \frac{\Gamma\left(\Delta-\frac{d}{2}\right)}{\Gamma(\Delta)} \int d^{d} x d^{d} x^{\prime} \frac{\rho(\vec{x}) \rho\left(\vec{x}^{\prime}\right)}{\left|\vec{x}-\vec{x}^{\prime}\right|^{2 \Delta}}
$$

Here we see the desirable properties of this formulation. The specific form of $\mathbf{A}$ gave, upon the removal of the cutoff, exactly the correct coefficient $-\left(\Delta-\frac{d}{2}\right)$ that was found in 13 to be necessary in order to obey Ward identities. The minus sign is important for the positivity of the CFT two-point function.

In the marginal case $\Delta=\frac{d}{2}$ the asymptotic behavior is given instead of (2.7) by:

$$
\phi(\vec{x}, z)=\left[\alpha(\vec{x}) z^{\frac{d}{2}} \log \left(\frac{z}{z_{0}}\right)+\beta(\vec{x}) z^{\frac{d}{2}}\right]\left(1+\mathcal{O}\left(z^{2}\right)\right)
$$

with $z_{0}$ a constant. An elegant property of this formulation is that due to the shift of the exponent in $\mathbf{A}$ with respect to $(2.6)(\gamma=\Delta+1)$ the above procedure remains true also in this marginal case6, with the result

$$
\lim _{z \rightarrow 0} \mathbf{A}\left(\vec{x}, \vec{x}^{\prime}\right)=\frac{-d \pi^{\frac{d}{2}}}{\Gamma\left(\frac{d}{2}+1\right)} \delta^{d}\left(\vec{x}-\vec{x}^{\prime}\right)
$$

The action one gets in this case is given in [8]. Moreover, scalar fields in $d$-dimensions obey a unitarity bound $\Delta \geq \frac{d-2}{2}$. In this formalism we see this bound to arise naturally as a bound on the applicability of the derivation leading to (2.18). Indeed unless $\Delta>\frac{d-2}{2}$

6 There is no divergence in (2.17) in the marginal case as can be seen by using the defining property of the Gamma function $0 \times \Gamma(0)=\Gamma(1)=1$. 
the coefficient in front of (2.17) diverges. The fact that in $\mathbf{A}$ the exponent is $\gamma=\Delta+1$ captures this property 月. This formalism is applicable also in the range $\frac{d}{2}>\Delta>\frac{d-2}{2}$, however in this range there are further subtelties [14, 15].

To summarize, this formalism reproduces correctly the usual $A d S / C F T$ results including some of its subtleties. It differs from the usual treatment of $A d S / C F T$ in that we use (2.10) instead of Dirichlet boundary condition. In this treatment the CFT source $\rho$ is also a source to the gravity field $\phi$ albeit with coupling only on the boundary of AdS. In the next section we show how to generalize to the case of multiple trace deformations of the dual $C F T$.

\section{AdS/CFT deformed by a Multiple-trace Operator.}

In this section we derive the effect of deforming the dual CFT with a Multi-trace operator. Start with an $A d S / C F T$ dual pair and denote the action of the CFT by $I_{0}\left[\varphi_{j}\right]$, where $\varphi_{j}$ are the fundamental fields of the CFT. Now consider, following [9], a general deformation of the CFT action

$$
I_{0} \rightarrow I_{W} \equiv I_{0}+W\left[\hat{\mathcal{O}}\left(\varphi_{j}\right)\right]
$$

by adding some function of the Single-trace operator

$$
\hat{\mathcal{O}} \equiv \operatorname{Tr}\left(\prod_{j} \varphi_{j}\right)
$$

Expanding the notation of (2.13) we write the classical gravity approximation to the $A d S / C F T$ correspondence as:

$$
Z[\rho]_{C F T} \equiv<e^{\int \rho \hat{\mathcal{O}}}>_{C F T}=\int D\left[\varphi_{j}\right] e^{-I_{0}\left[\varphi_{j}\right]} e^{\rho O\left(\varphi_{i}\right)} \simeq \int D[\phi] e^{-\mathcal{S}_{g r}[\phi ; \rho]} \sim e^{-\mathcal{S}_{g r}\left[\phi_{\rho} ; \rho\right]},
$$

where as before $\rho$ is a classical source, $\langle\ldots\rangle_{C F T}$ stands for $\int D\left[\varphi_{j}\right] e^{-I_{0}\left[\varphi_{j}\right]}(\ldots)$ and we use the notation $\hat{\mathcal{O}}$ for the quantum operator acting in the CFT Hilbert space and $O$ for

7 This bound is also necessary for the expansion (2.7) to give the leading terms when $z \rightarrow 0$. Indeed the $\mathcal{O}\left(z^{2}\right)$ corrections are smaller when $\Delta<\frac{d}{2}$ if $d-\Delta<\Delta+2$.

8 The case $\Delta=\frac{d-2}{2}$ corresponds to a free CFT field. It is thus not surprising that we do not see this behavior in the gravity approximation. 
the usual (commutative) function which enters in the corresponding path integral. To find the gravity action dual to the deformed CFT we proceed as follows:

$$
\begin{aligned}
Z_{C F T}^{W}[\rho] & \equiv<e^{\int \rho \hat{\mathcal{O}}}>_{C F T}^{W} \equiv<e^{\int \rho \hat{\mathcal{O}}-W[\hat{\mathcal{O}}]}>_{C F T}= \\
& =\int D\left[\varphi_{j}\right] e^{-I_{0}\left[\varphi_{i}\right]-W\left[O\left(\varphi_{i}\right)\right]+\rho O\left(\varphi_{i}\right)}=\int D\left[\varphi_{i}\right] e^{-I_{0}} e^{-W[O]} e^{\rho O}= \\
& =\int D\left[\varphi_{i}\right] e^{-I_{0}} e^{-W\left[\frac{\delta}{\delta \rho}\right]} e^{\rho O}=e^{-W\left[\frac{\delta}{\delta \rho}\right]} \int D\left[\varphi_{i}\right] e^{-I_{0}} e^{\rho O}= \\
& =e^{-W\left[\frac{\delta}{\delta \rho}\right]}<e^{\int \rho \hat{\mathcal{O}}}>_{C F T}=e^{-W\left[\frac{\delta}{\delta \rho}\right]} \int D[\phi] e^{-\mathcal{S}_{g r}[\phi ; \rho]}= \\
& =e^{-W\left[\frac{\delta}{\delta \rho}\right]} \int D[\phi] e^{-\left(\mathcal{S}_{0}[\phi]+\epsilon^{-\Delta} \phi \mathbf{A} \rho\right)}=\int D[\phi] e^{-\mathcal{S}_{0}[\phi]} e^{-W\left[\frac{\delta}{\delta \rho}\right]} e^{-\epsilon^{-\Delta} \phi \mathbf{A} \rho}= \\
& =\int D[\phi] e^{-\mathcal{S}_{0}[\phi]} e^{-W\left[-\epsilon^{-\Delta} \mathbf{A} \phi\right]} e^{-\epsilon^{-\Delta} \phi \mathbf{A} \rho}=\int D[\phi] e^{-\mathcal{S}_{g r}[\phi ; \rho]} e^{-W\left[-\epsilon^{-\Delta} \mathbf{A} \phi\right]} \equiv \\
& \equiv \int D[\phi] e^{-\mathcal{S}_{g r}[\phi ; \rho, W]} \sim e^{-\mathcal{S}_{g r}\left[\phi_{c l} ; \rho, W\right]} .
\end{aligned}
$$

A crucial assumption in this derivation is that (2.10) is not a boundary condition but a boundary equation of motion, i.e. the measure $D[\phi]$ does not depend on $\rho$.

To summarize the computation, we found that at a finite cutoff the gravity action dual to the CFT deformed as in (3.1) is:

$$
\mathcal{S}_{g r}[\phi ; \rho, W] \equiv \mathcal{S}_{g r}[\phi ; \rho]+W\left[-\int_{\partial} d^{d} x^{\prime} \epsilon^{-\Delta} \mathbf{A}\left(\vec{x}, \vec{x}^{\prime}\right) \phi\left(\vec{x}^{\prime}, z\right)\right]
$$

From here we proceed to find the boundary relation in the same way as for (2.11):

$$
\begin{aligned}
\delta \mathcal{S}_{g r}[\phi ; \rho, W] & =\int_{\mathcal{B}} d^{d+1} x \sqrt{g} \delta \phi\left[-\nabla^{2}+m^{2}\right] \phi \\
& -\int_{\partial} \epsilon^{-\Delta} \delta \phi\left(z^{\Delta-d}\left(z \partial_{z}-\Delta\right) \phi-\mathbf{A} \rho+\mathbf{A} \frac{\delta W[\psi]}{\delta \psi}\right),
\end{aligned}
$$

where we denoted the argument of $W$ by $\psi$ which in the condensed notation introduced before is given by:

$$
\psi \equiv-\int_{\partial} d^{d} x^{\prime} \epsilon^{-\Delta} \mathbf{A}\left(\vec{x}, \vec{x}^{\prime}\right) \phi\left(\vec{x}^{\prime}, z\right) \equiv-\epsilon^{-\Delta} \mathbf{A} \phi
$$

Since the path integral in (3.4) has no boundary condition, a classical solution must obey, apart from the bulk equation of motion, also the following boundary equation of motion:

$$
\left.z^{\Delta-d}\left(z \partial_{z}-\Delta\right) \phi\right|_{\partial}=\mathbf{A}\left(\rho-\frac{\delta W[\psi]}{\delta \psi}\right) .
$$


This is the main result of this work. It shows how the deformation of the dual CFT by a Multi-trace operator changes the action and the boundary equation of motion in gravity.

The generalization to a function of many variables $W\left[\hat{\mathcal{O}}_{1}, \ldots, \hat{\mathcal{O}}_{n}\right]$ is straightforward. The action becomes:

$$
\mathcal{S}_{g r}\left[\phi_{1}, \ldots, \phi_{n} ; \rho, W\right] \equiv \sum_{i=1}^{n} \mathcal{S}_{g r}\left[\phi_{i} ; \rho_{i}\right]+W\left[\psi_{1}, \ldots, \psi_{n}\right],
$$

where $\psi_{i} \equiv-\epsilon^{-\Delta_{i}} \mathbf{A}_{i} \phi_{i}$ and with the following boundary equation of motion for each field:

$$
\left.z^{\Delta_{i}-d}\left(z \partial_{z}-\Delta_{i}\right) \phi_{i}\right|_{\partial}=\mathbf{A}_{i}\left(\rho_{i}-\frac{\delta W\left[\psi_{1}, \ldots, \psi_{n}\right]}{\delta \psi_{i}}\right) .
$$

In the specific example discussed in [8]:

$$
W[\hat{\mathcal{O}}]=\frac{\tilde{h}}{2} \hat{\mathcal{O}}^{2},
$$

we get the following deformed boundary equation of motion:

$$
\left.z^{\Delta-d}\left(z \partial_{z}-\Delta\right) \phi\right|_{\partial}=\mathbf{A}\left(\rho+\tilde{h} \epsilon^{-\Delta} \mathbf{A} \phi\right),
$$

reproducing the result of [8] which was obtained using an auxiliary field method.

In [9] Witten reasoned based on a matrix model analogy that the boundary relation in $A d S$ gravity corresponding to a Multi-trace deformation of the form (3.1) is

$$
\alpha=\frac{\delta W[\beta]}{\delta \beta},
$$

with $\alpha, \beta$ given by (2.7). This matches with the results presented above since when the cutoff is removed the LHS of (3.8) essentially picks out the $\alpha$ up to a coefficient (see (2.9)) while from (2.17) the RHS of (3.8) becomes $\frac{\delta W[\psi]}{\delta \psi}=\frac{\delta W[\beta]}{\delta \beta}$.

\section{Discussion and Summary}

\subsection{Marginality and General Covariance.}

If $W\left[\hat{\mathcal{O}}_{1}, \ldots, \hat{\mathcal{O}}_{n}\right]$ is a marginal deformation in the CFT then it contains only monomial terms of dimension $d$. Correspondingly $W\left[-\epsilon^{-\Delta_{1}} \mathbf{A}_{1} \phi_{1}, \ldots,-\epsilon^{-\Delta_{n}} \mathbf{A}_{n} \phi_{n}\right]$ on the gravity side will have only terms of the form $\epsilon^{-d} \prod \phi_{j}$. In Poincare coordinates the boundary volume element is $\sqrt{\widehat{g}}=\epsilon^{-d}$. Thus the deformation of the gravity action can be written in a general covariant way. From (2.6) we see that this boundary volume element is needed in order to get a finite boundary integral in the limit $\epsilon \rightarrow 0$.

9 This is so because when $\epsilon \rightarrow 0 \psi \rightarrow \epsilon^{d-2 \Delta} \alpha(\vec{x})+\beta(\vec{x})$ (up to coefficients) and thus $\frac{\delta \psi(\vec{x})}{\delta \beta(\vec{y})}=$ $\delta^{d}(\vec{x}-\vec{y})$. The extra $\rho$ in $(3.8)$ is just a matter of difference in conventions with respect to [9] since we singled out the linear term in $W$. 


\subsection{Renormalizability.}

The way in which the deformation term $W$ enters both the CFT action (3.1) and the dual gravity action (3.5) suggests that any renormalization procedure applied on the CFT side by adding local counter-terms translates straightforwardly to the gravity side. Thus if the CFT action must be supplemented with a counter-term $I+W[\hat{\mathcal{O}}] \rightarrow I+$ $(W[\hat{\mathcal{O}}]+R[\hat{\mathcal{O}}])$ then following the same steps as in (3.4) we see that the gravity action acquires a corresponding boundary term $\mathcal{S} g r+W[\psi] \rightarrow \mathcal{S} g r+(W[\psi]+R[\psi])$. This fact was demonstrated explicitly in [8] for the case (3.11).

\subsection{Boundary condition vs boundary equation of motion.}

An important theme discussed in this note is the issue of boundary condition versus boundary equation of motion in $A d S / C F T$. In the usual correspondence described in section 2 it seems that there is not much of a difference whether one chooses Dirichlet boundary condition or (2.10). Furthermore, in the classical limit it is more a matter of semantics whether one thinks of (2.5) as a boundary condition constraining the phase space of allowed quantum fluctuations or as a boundary equation of motion standing on an equal footing with the bulk equation of motion. It is therefore interesting that when one considers Multi-trace deformations the natural choice turns out to be (2.10). The derivation (3.4) clearly supports the interpretation of (2.10) as a boundary equation of motion and not as a boundary condition. The work done in [8] also supports this interpretation, since although $A d S / C F T$ was formulated there with a boundary condition, the result was that after a Double-trace deformation one got (3.5) without a boundary condition 10 . It thus looks more natural if the "usual" case is formulated in the same way as the one with Multi-trace deformations. We conclude that our analysis seems to give non-trivial information about the quantum gravity side of $A d S / C F T$. Of course, the claim that $A d S / C F T$ "has no boundary condition" raises some questions regarding conservation of charges etc. Also, it is not entirely clear if the distinction is really so sharp. Indeed the only fluctuations around the classical solution which contribute to the path integral are the ones which behave near the boundary like $\delta \phi \sim z^{\kappa}$ with $\kappa>\frac{d}{2}$ or $\kappa=\frac{d}{2}-\sqrt{\frac{d^{2}}{4}+m^{2}}$. At least when $\Delta>\frac{d}{2}$ fluctuations of the first kind automatically satisfy (2.10) when $\epsilon=0$, while those of the second kind are infinitely supressed in the path integral. It thus seems that the difference between the two options before the deformation vanishes in this case when one removes the

10 The boundary conditions where explicitly integrated over in [8]. 
cutoff11. A last remark is that the string theory description of spacetimes with boundaries is an interesting and subtle problem; see e.g. the discussion in [8].

\section{Acknowledgments}

We would like to thank R. Argurio, J.L.F. Barbon, D.S. Berman, M. Dine, S. Elitzur, G. Ferretti, A. Giveon, A. Hanany, Y. Oz, A. Petkou, E. Rabinovici for useful discussions. We owe special thanks to M. Berkooz and O. Aharony for advising us and for a critical reading of the manuscript. A.Sh thanks the theory devision in CERN and the string group in Chalmers university, Gothenburg, for hospitality during the final stages of this work. A.Sh is supported by a Clore fellowship. This work is supported in part by BSF - American-Israel Bi-National Science Foundation, the Israel Academy of Sciences and Humanities - Centers of Excellence Program, the German-Israel Bi-National Science Foundation, the European RTN network HPRN-CT-2000-00122.

11 When $\epsilon=0$ the depth of the well around the boundary behavior of the classical solution becomes infinite. 


\section{References}

[1] J. Maldacena, "The large $N$ limit of superconformal field theories and supergravity," Adv. Theor. Math. Phys. 2 (1998) 231 ; Int. J. Theor. Phys. 38 (1998) 1113 ; hepth/9711200.

[2] E. Witten, "Anti-de Sitter space and holography," Adv. Theor. Math. Phys. 2 (1998) 253 ; hep-th/9802150.

[3] S. S. Gubser, I. R. Klebanov and A. M. Polyakov, "Gauge theory correlators from non-critical string theory," Phys. Lett. B 428 (1998) 105 ; hep-th/9802109.

[4] O. Aharony, S. S. Gubser, J. Maldacena, H. Ooguri and Y. Oz, "Large N field theories, string theory and gravity," Phys. Rept. 323 (2000) 183 ; hep-th/9905111.

[5] E. D'Hoker and D. Z. Freedman, "Supersymmetric gauge theories and the AdS/CFT correspondence," ; hep-th/0201253.

[6] O. Aharony, M. Berkooz and E. Silverstein, 'Multiple-trace operators and non-local string theories," JHEP 0108 (2001) 006 ; hep-th/0105309.

[7] O. Aharony, M. Berkooz and E. Silverstein, "Non-local string theories on AdS(3) x $\mathrm{S}^{* *} 3$ and stable non-supersymmetric backgrounds," ; hep-th/0112178.

[8] M. Berkooz, A. Sever and A. Shomer, "Double-trace deformations, boundary conditions and spacetime singularities," ; hep-th/0112264.

[9] E. Witten, "Multi-trace operators, boundary conditions, and AdS/CFT correspondence," ; hep-th/0112258.

[10] P. Minces and V. O. Rivelles, "Energy and the AdS/CFT correspondence," JHEP 0112 (2001) 010 ; hep-th/0110189.

[11] P. Minces, "Multi-trace operators and the generalized AdS/CFT prescription," ; hepth/0201172.

[12] W. Muck, "An improved correspondence formula for AdS/CFT with multi-trace operators," Phys. Lett. B 531 (2002) 301 ; hep-th/0201100.

[13] D. Z. Freedman, S. D. Mathur, A. Matusis and L. Rastelli, "Correlation functions in the CFT $(d) / \operatorname{AdS}(d+1)$ correspondence," Nucl. Phys. B 546 (1999) 96 ; hepth/9804058.

[14] I. R. Klebanov and E. Witten, "AdS/CFT correspondence and symmetry breaking," Nucl. Phys. B 556 (1999) 89 ; hep-th/9905104.

[15] P. Breitenlohner and D. Z. Freedman, "Stability In Gauged Extended Supergravity," Annals Phys. 144 (1982) 249. 